\title{
Real-Time State Estimation of the EPFL-Campus Medium-Voltage Grid by Using PMUs
}

Marco Pignati, Miroslav Popovic, Sergio Barreto, Rachid Cherkaoui, German Dario Flores, Jean-Yves Le Boudec, Maaz Mohiuddin, Mario Paolone, Paolo Romano, Styliani Sarri, Teklemariam Tesfay, Dan-Cristian Tomozei, Lorenzo Zanni

DESL \& LCA2 labs, EPFL, Switzerland

\begin{abstract}
We describe the real-time monitoring infrastructure of the smart-grid pilot on the EPFL campus. We experimentally validate the concept of a real-time state-estimation for a $20 \mathrm{kV}$ active distribution network. We designed and put into operation the whole infrastructure composed by the following main elements: (1) dedicated PMUs connected on the medium-voltage side of the network secondary substations by means of specific current/voltage transducers; (2) a dedicated communication network engineered to support stringent time limits and (3) an innovative state estimation process for real-time monitoring that incorporates phasor-data concentration and state estimation processes. Special care was taken to make the whole chain resilient to cyber-attacks, equipment failures and power outages. The achieved latency is within $65 \mathrm{~ms}$. The refresh rate of the estimated state is $20 \mathrm{~ms}$. The real-time visualization of the state estimator output is made publicly available, as well as the historical data (PMU measurements and estimated states). To the best of our knowledge, the work presented here is the first operational system that provides low-latency real-time stateestimation by using PMU measurements of a real active distribution network.
\end{abstract}

Index Terms--Active distribution networks, communication network, phasor measurement units, real-time monitoring, state estimation, synchrophasors.

\section{INTRODUCTION}

The evolution of distribution networks towards the socalled active distribution networks (ADNs) requires the availability of suitable distributed/centralized processes designed to achieve specific operation objectives (e.g., IEEE Standards: 1547.4, 1547.6, 2030): (i) optimal voltage control, (ii) line congestion management, (iii) fault detection and location, (iv) post-fault management, (v) local load balance, (vi) network losses minimization.

These operations are significantly improved if the system state is known. In this respect, typical refresh rates of the existing SCADA-based state estimation (SE) processes are in the order of a few minutes/seconds, whereas the abovementioned functionalities' time frames are between few hundreds of milliseconds (fault management) to a few seconds (voltage control and line congestions in presence of highlyvolatile renewables). In view of the typical streaming rates of PMUs (i.e., in the order of some tens of synchrophasors per second, as described in IEEE C37.118 standard), there is, in principle, the possibility of developing sub-second SE processes that can enable, the definition of new protection and control schemes for ADNs.

However, to the best of the our knowledge, none of the contributions listed in Section II below, has proposed and experimentally validated the timing latency and accuracy of a full real-time 3-phase SE process deployed in a real ADN. In this respect, this work contributes the following: (a) describes specific PMUs embedding dedicated synchrophasor estimation processes designed for ADN applications, (b) describes the formulation of a 3-phase discrete Kalman filter (DKF) SE algorithm previously developed by the Authors and used in the context of this paper, (c) illustrates a dedicated communication network engineered to support stringent time limits and, finally, (d) describes a dedicated process incorporating the phasor-data concentration and stateestimation functionalities.

\section{STATE OF THE ART}

The literature has produced a number of contributions discussing the methodological aspects related to the 3-phase state estimation of ADNs. Classic methods used for the solution of the 3-phase SE problem are usually based on the use of the weighted least squares (WLS) method (e.g., [1], [2]). In [3] Dzafic et al. present a new RTSE algorithm based on a weighted grouping of measurements. Methods based on the use of current measurements that use rectangular coordinates (e.g., [4]-[7]) have been proposed too. In [8] $\mathrm{Li}$ used a 3-phase WLS to make an assessment of the SE accuracy as a function of the load-error correlation, the availability of RT measurements, pseudo-measurement errors and measurement placement. The work presented in [9] illustrates a comparison, in terms of estimation accuracy and computation time, between a 1-phase equivalent and a 3-phase WLS-based SE for both transmission and distribution networks. In [10], the authors proposed the use of RT measurements with the forecasted loads or pseudomeasurements to improve the accuracy of SE, and they show the impact of power flow and current measurements on baddata identification. 
State estimation with PMU measurements is also vastly treated in the literature. The deployment, however, of such technology in real networks has not yet been largely experienced, especially in the distribution networks. The implementation, testing and performance assessment of a phasor-assisted state estimation for transmission networks is provided in [11]. A 3-phase linear-state estimation using phasor measurements only is presented in [12]. This is the first real implementation able to provide the 3-phase system's state with a refresh rate up to 30 times per second. However, the paper presents the results obtained on a transmission network and does not mention the possibility of extending such a concept to distribution networks. Furthermore, this contribution has not assessed the latency of the process as well as the accuracy of the SE.

Few works present a state-estimation process capable of operating at the distribution level. In [13] inputs to the state estimator are provided by analog measurements and by pseudo-measurements provided by load profiles instead of PMUs. Microgrid at IIT project (http://www.iitmicrogrid.net/) does not comprise real-time state estimation. The work presented in [14] reported state estimation only as a future work. In addition, the communication network that is described is not dedicated nor fully controlled, making the whole system unreliable. Therefore, to the best of our knowledge, the work presented in this paper is the very first deployed real-time state estimator for distribution-networks.

\section{THE SYSTEM ARCHITECTURE}

Our smart grid infrastructure is deployed on the electrical network of the EPFL campus. It is a particularly challenging distribution network where all the peculiarities of ADN are stressed. The lines are short (most of them below $100 \mathrm{~m}$ ), and the load demand is largely variable in function of the hour of the day and the weather conditions. Moreover, active power injections are present as $2 \mathrm{MW}$ of photovoltaic panels are installed together with $6 \mathrm{MW}$ of combined heat and power generation units. These conditions, and the large use of power electronics, heavily affect the voltage and current profiles which makes the EPFL campus a challenging test bed for the developed infrastructure. Figure 1 depicts the map of the campus with the feeder that is equipped with PMUs and for which the state estimation is performed.

The whole process starts with the sensors that are connected to the medium-voltage side of the transformers. Voltage and current scaled waveforms are then fed to the PMUs in order to estimate the synchrophasors. Specific characteristics of sensors and PMUs are given in Section III.A. PMUs encapsulate UDP datagrams according to IEEE C37.118.2-2011. Datagrams are transmitted over a secured and dedicated communication network (Sections III.B and III.C). A dedicated low-latency PDC has been developed (Section III.D). It takes care of decapsulation, time-alignment and the replacement of missing measurements in order to feed the real-time SE with a consistent and complete set of data. The SE is based on Kalman filtering technique and it is developed in LabVIEW (see Section III.E). The measurements sent by the PMUs and the state estimator outputs are saved on our servers and made publicly available (Section IV).

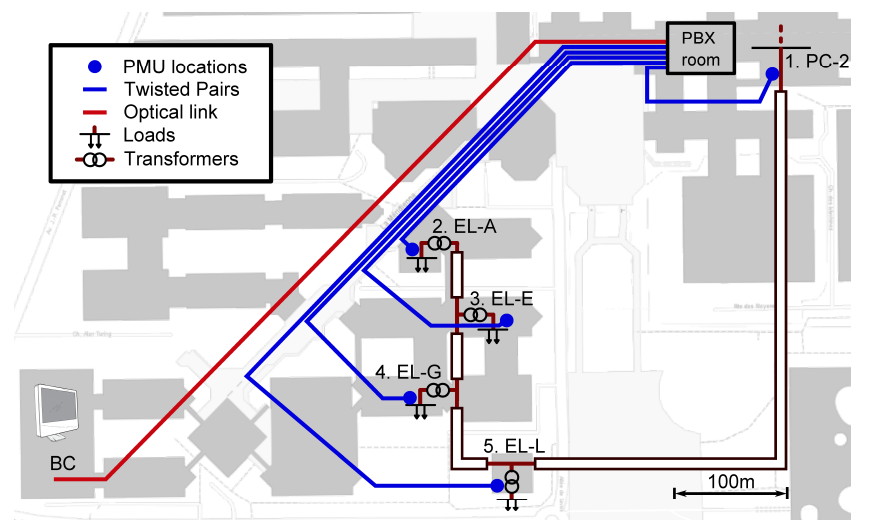

Figure 1 - EPFL smart-grid infrastructure.

\section{A. Phasor Measurement Units}

Phasor measurement unit (PMU) technology is widely considered as the most advanced metering infrastructure for power systems, because it allows for synchronized and fast measurements of frequency, amplitude and phase of the power-system waveforms. The requirements defined by the IEEE Std. C37.118.1-2011 and its latest amendment IEEE Std. C37.118.1a-2014 were originally intended for PMUs deployed in transmission networks. In order to allow for the use of this technology in ADNs, PMUs need to satisfy severe requirements in terms of phase accuracy, harmonic rejection and performances during power system dynamics [15].

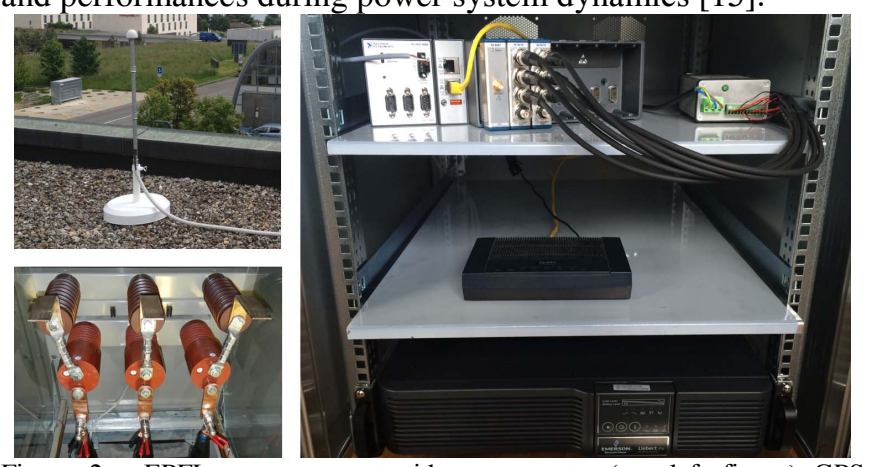

Figure 2 - EPFL-campus smart-grid components: (top left figure) GPS antenna, (bottom left figure) Altea CVS-24 current and voltage sensors and (figure on the right) rack containing the PMU (top shelf), the SHDSL modem (middle shelf) and the UPS (bottom shelf).

In this respect we have adopted the PMUs discussed in [16] where the Authors have presented a novel synchrophasor estimation algorithm that improves the performances of the so-called interpolated-DFT method by means of a specific compensation scheme for the spectral interference produced by the negative image of the spectrum. In [16] the synchrophasor estimation algorithm is presented, together with its deployment in a National Instruments CompactRIO 9068, composed by a reconfigurable Artix-7 FPGA and a dual-core ARM Cortex-A9 processor and equipped with a customized Linux-RT OS. The chassis has been equipped with a stationary GPS unit (NI-9467) for the synchronization to the UTC-time and two analog input modules (NI-9215) characterized by an input range of $\pm 10 \mathrm{~V}$ and a sampling frequency of $50 \mathrm{kHz}$ (see Figure 2). The synchrophasor estimation algorithm has been fully deployed on the FPGA 
target only, letting the CPU free to perform any other task such as the streaming of the estimated quantities based on the protocol specified by the standard. The experimental validation presented in [16] demonstrates that the developed PMU prototype exhibits peculiar characteristics that enable its use in ADNs. In particular, this PMU satisfies every requirement defined in the standard for class-P PMUs.

In order to interface the PMU analog input modules with the power-system waveforms (i.e., 3-phase nodal voltages and nodal injected currents), the high-voltage/current signals need to be transformed to low voltage signals with the minimum amplitude/phase distortion possible. In this respect, an improved version of the Altea CVS-24 [17] was selected as the one that offers the best performances at a reasonable cost and size. These sensors are 0.1-class compliant (Figure 2 shows a sensor installation). The connection between the sensors output and the PMU has been made by means of dedicated shielded-cables of equal fixed length for all the substations. The PMU synchronization is achieved by means of dedicated GPS installations for each electrical substation (see Figure 2). The connection between the GPS antenna mounted on the rooftop and the PMU was designed using RG213 cables in order not to attenuate the GPS signal also with more than 100 meters of cables. The cable length delay was suitably compensated on the PMU side.

\section{B. Communication Network}

We built a dedicated communication network for security and robustness (the campus intranet is not secured in case of power outages). We use IPv6 rather than IPv4 in order to prevent future transition issues. We avoided expensive cabling deployments by re-using existing twisted pair cables, originally installed for telephony. These cables are passive and are star-wired from a central point, the "PBX room", where backup power is available. Communication over twisted pair cables uses the single-pair high-speed digital subscriber line (SHDSL) technology, as these cables are too long for Ethernet. Traffic from all PMUs is concentrated at the SHDSL concentrators (called DSLAMs) located in the PBX room. This would also be the natural place to locate the PDC and SE machines; however, we have only very restricted access to it. Thus, we had to place the PDC and SE machines in a more convenient location; for communications from the PBX room to PDC, we had to use more expensive optical fibers at $100 \mathrm{Mb} / \mathrm{s}$, as the bitrate of SHDSL $(2 \mathrm{Mb} / \mathrm{s})$ is not sufficient here. The whole network is resilient to up to 8 hours of power outage; it is traffic-engineered to ensure enough capacity for the generated traffic.

The entire communication network is in fact duplicated, as seen in Figure 3. We developed an IP version of the parallel redundancy protocol, called IPRP [18]; it takes care of duplicating UDP packets (at PMUs) and removing duplicates (at the PDC). This provides 0-ms repair of packet losses. We implemented IPRP as a transport layer solution in the Linux operating systems of the PMUs and PDC; this has the benefit of not requiring any changes to any PMU/PDC applications or to any network devices.

Raw measurements and state estimator outputs are made public through a web interface ${ }^{1}$. Every stored file contains one hour of data; it is a self-explanatory text file that is digitally signed.

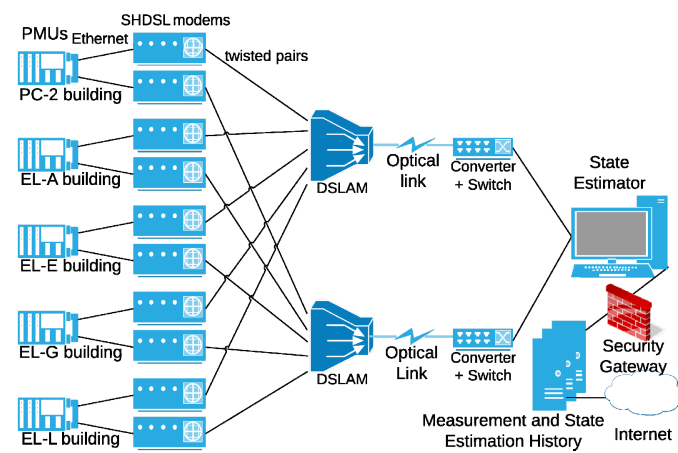

Figure 3 - EPFL-campus smart-grid communication network.

\section{Cyber-security}

We have also put security mechanisms into place to ensure that the ICT infrastructure is resilient to insider and outsider cyber-attacks. The security mechanisms we implement guarantee that access to all devices in the ADN is limited only to authorized personnel. Each authorized personnel is assigned separate user credentials; everyone is held accountable for their activities in the network. Accountability is enforced by implementing a logging mechanism to record each and every activity a user performs and by analyzing the log data to identify suspicious activities.

We have also implemented network-access control mechanisms to prevent an outsider from gaining access to the AND that uses a malicious (rogue) device. All devices directly connected to the ADN are authenticated using their credentials (digital certificates) before they start any sort of communication with any device in the network. The digital certificates are also used to secure the communication between the field devices (PMU's) and the PDC. DTLS is used for application-layer (end-to-end) secure communication. We also implement MACSec for hop-by-hop security in order to ensure that bogus traffic injected by a rogue device is discarded at the next switch. This prevents DoS attacks because such traffic does not propagate beyond the first link where the traffic is injected.

The ADN's communication infrastructure is also physically separate from the rest of the campus' public communication infrastructure. There is only minimal communication with the public network in order to publish the synchrophasor data, as well as the SE output, to the outside world. This communication passes through a single tightly secured interfacing point (security gateway) that opens only the required ports (Figure 3 ). This security gateway serves as a protective barrier by effectively shielding the ADN from any incoming attacks from the public network.

\section{Phasor-Data Concentrator}

The phasor-data concentrator (PDC) collects synchrophasor data and other quantities (i.e., frequency, rateof-change-of-frequency, nodal injected/absorbed powers,

\footnotetext{
${ }^{1}$ http://smartgrid.epfl.ch/
} 
etc.) estimated by the PMUs and transmits the information to other applications such as visualization tools or state estimator (see Section III.E). The PDC deployed on the EPFL campus was fully developed by the Authors in LabVIEW and it is hosted, together with the state estimation, on a dedicated workstation. Among other functionalities, the PDC is able to communicate with the PMUs and decapsulates their IEEE C37.118.2 datagrams. The synchrophasors are aggregated and time-aligned in a circular buffer, according to their timestamps. A subset of these measurements is then pushed to the state estimator with minimum time latency which distribution is shown in Section IV. Indeed, when dealing with real communication networks and devices, measurements can be delayed due to non-determinism in the data streaming, network traffic, different reporting rates among the devices, etc. In this case, there is no way to determine the exact amount of time the data packet takes to be received. For this reason, an adaptive algorithm was developed that determines, based on an event-timeout, the amount of time each dataset has to actively wait for the rest of phasors with the same timestamp. The timeout expires with the arrival of any measurement with a more recent timestamp compared to the one that the buffer is currently waiting for. When the event is triggered, the current dataset is forwarded to the SE application without waiting for the eventual missing measurements to arrive. This solution always ensures that the available measurements will be forwarded in an acceptable time range $(20 \mathrm{~ms}$ in case of PMUs streaming at $50 \mathrm{fps}$ ) thus increases the determinism of the process.

Finally, we note that the machine hosting the PDC and SE is GPS synchronized, hence we are able to identify and solve eventual bottlenecks in the whole chain that might result in an increased total latency (see Section IV).

\section{E. State Estimation}

The state estimator receives the data from the PDC and estimates the system state $\mathbf{x} \in \mathbb{R}^{3 \times(2 n)}$, defined as

$$
\mathbf{x}=\left[V_{1, r}^{a, b, c}, \cdots, V_{n, r}{ }^{a, b, c}, V_{1, i m}{ }^{a, b, c}, \cdots, V_{n, i m}{ }^{a, b, c}\right]^{T}
$$

Here $n$ is the number of buses and $V_{i, r}{ }^{a, b, c}, V_{i, i m}{ }^{a, b, c}$ are the 3phase real and imaginary parts of the voltage phasor at bus $i$. Two different SE methods were implemented in the context of this project, i.e., the classic weighted least squares (WLS) and the discrete Kalman filter (DKF). However, after assessing off-line the two SE algorithms discussed in [19], we find that the latter has provided more accurate estimations; therefore DKF has been finally adopted over WLS.

As known, DKF with no control input addresses the problem of estimating the state of a discrete-time system process governed by the following set of linear stochastic equations (e.g., [20]):

$$
\begin{gathered}
\mathbf{x}_{k}=\mathbf{x}_{k-1}+\mathbf{w}_{k-1} \\
\mathbf{z}_{k}=\mathbf{H} \mathbf{x}_{k}+\mathbf{v}_{k}
\end{gathered}
$$

where:

- $\quad k$ is the time-step index;

- $\quad \mathbf{w} \in \mathbb{R}^{3 \mathrm{x}(2 n)}$ represents the process noise, $p(\mathbf{w}) \sim N(0, \mathbf{Q})$;

- $\quad \mathbf{z} \in \mathbb{R}^{m}$ represents the set of measurements;

- $\quad \mathbf{v} \in \mathbb{R}^{m}$ represents the measurement noise, $p(\mathbf{v}) \sim N(0, \mathbf{R})$;

The adopted DKF-SE is suitable for 3-phase systems and relies only on nodal synchrophasor measurements provided by PMUs; therefore $\mathbf{z}$ depends linearly - through matrix $\mathbf{H}$ - on the system state $\mathbf{x}$ and includes nodal voltage and injected current phasors. As a consequence, $\mathbf{H}$ consists of constant elements, namely: zeroes and ones for the part that is related to the nodal voltage measurements, and the admittance matrix elements for the part related to the measured injected currents.

Equation (2) represents the DKF process model, assumed to be an autoregressive integrated moving average (ARIMA) model of order $(0,1,0)$ (see [19]).

The two noise-covariance matrices $\mathbf{R}$ and $\mathbf{Q}$ influence significantly the DKF-SE accuracy: $\mathbf{R}=\operatorname{diag}\left(\sigma_{1}^{2}, \ldots, \sigma_{\mathrm{m}}{ }^{2}\right)$ represents the accuracies of the measurement devices (PMU + sensors); each term $\sigma_{\mathrm{i}}^{2}$ is inferred by analyzing the measurement noise at the PDC side.

$\mathbf{Q}$ represents the uncertainty introduced by the process model to predict the system state and it is not trivial to determine. Therefore, in this paper, we assess $\mathbf{Q}$ at every timestep as proposed by Method \#2 in [19].

\section{PERFORMANCE EVALUATION}

In this section, we present the performances of the entire monitoring system by making reference to a typical dynamic observed on the EPFL campus within a time window of $5 \mathrm{~min}$. The state estimation is performed by using the following measurement set: voltage phasors in bus PC-2 and injected current phasors in all the buses.

Figure 4 shows the profiles of the voltage magnitude and phase at bus EL-E, phase C, within a time window of 10s. It comprises the measured and the estimated states (similar results are obtained for the other buses and phases). The measurement noise filtering action of the KF can be clearly observed.

In order to evaluate the SE accuracy, we compare the results obtained by two state estimators. The first one uses the measurement set defined before (i.e., voltage phasors in bus PC-2 and injected current phasors in all the other buses), and the second one uses all voltage and current phasors measured by PMUs in all the nodes. The state estimated by the latter is assumed to be the reference. Indeed, it relies on a measurement set with almost two times the amount of information compared to the first. The distributions of the differences of the estimated nodal injected/absorbed powers of these estimators is shown in Figure 5 with respect to the whole 5 min time window. The order of magnitude of the errors of nodal absorbed/injected powers are in the range of hundreds of W/VAr. Considering that the typical values of these quantities in the considered network are in the range of a few $\mathrm{kW} / \mathrm{kVAr}$ to tens of $\mathrm{kW} / \mathrm{kVAr}$, we obtain an overall accuracy of the estimated state in the range of few percents. 

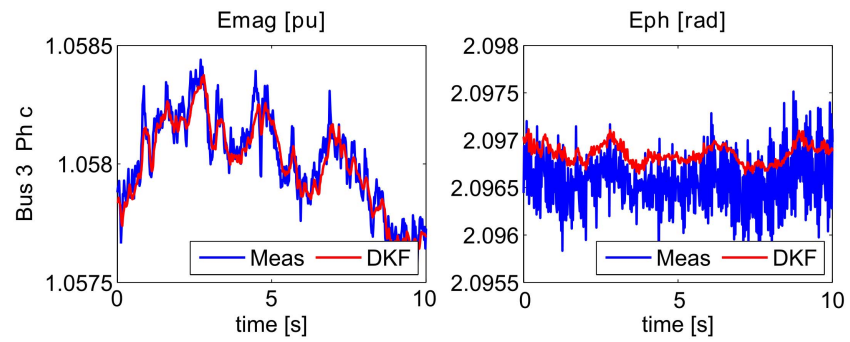

Figure 4 - Magnitude and phase of the phase-to-ground voltage of bus EL-E phase C: comparison between measured and estimated states (line-to-line base voltage equal to $20 \mathrm{kV}$ ).
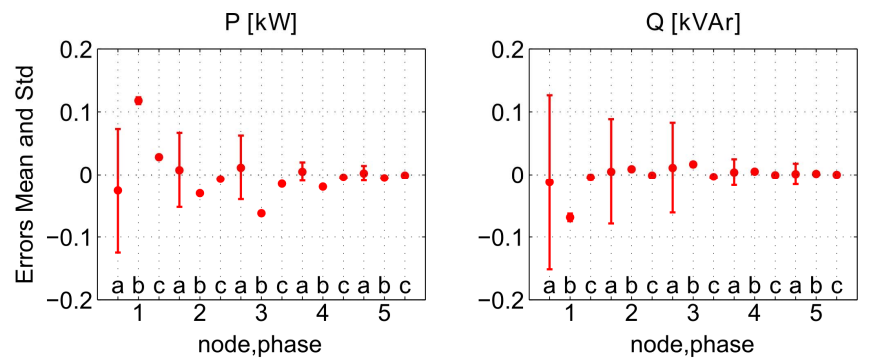

Figure 5 - P, Q estimation errors: mean and standard deviation.

Figure 6 shows the latency of the overall process expressed as cumulative distribution functions (CDFs) of time differences between specific sections of the above-described chain. As it can be seen, the PMU data sampling and synchrophasor estimation $\left(t_{1}\right.$ and $\left.t_{2}\right)$ are deterministic as they are performed on an FPGA [16]. The data encapsulation $\left(t_{3}\right)$ is the most non-deterministic contribution to latency. A delay of approx. $1.5 \mathrm{~ms}$ is added by data streaming $\left(t_{4}\right)$. The buffering in the PDC $\left(t_{5}\right)$ allows us to reduce the non-determinism added by the encapsulation. The overall latency has a mean value of $61 \mathrm{~ms}$ with a standard deviation of $1.8 \mathrm{~ms}$.

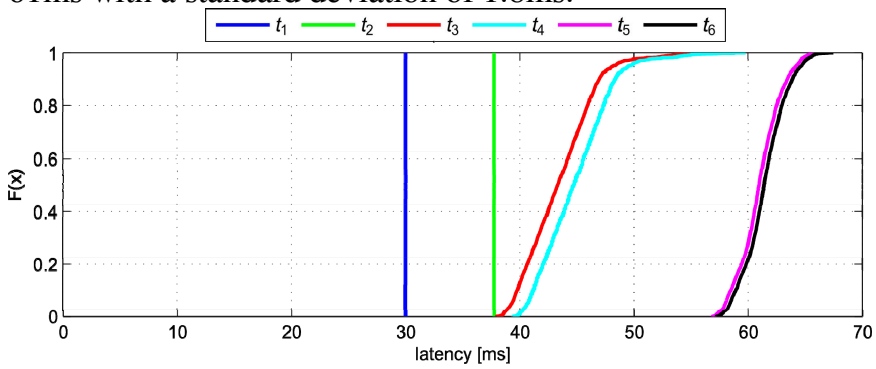

Figure 6 - CDF of time latencies.

\section{CONCLUSION AND FUTURE WORK}

The paper has illustrated the architecture for the real-time monitoring of the medium voltage network of the EPFL campus. This network was selected because it contains all the peculiarities of an ADN, namely short lines, high-volatile load demand, active power injections due to $2 \mathrm{MW}$ photovoltaic panels, and $6 \mathrm{MW}$ of combined heat and power generation units. The monitoring system that we built estimates the network state by using only PMU measurements in order to achieve extremely low latencies and high state estimation frame rates. To the best of our knowledge, this contribution is the first one to illustrate, and experimentally validate, the accuracy and time latency of a full real time 3-ph SE process deployed into a real ADN. The performances of the developed system appear compatible for its coupling with real-time protection and control functionalities expected to be developed for ADNs.

\section{REFERENCES}

[1] A. P. Meliopoulos, and F. Zhang, "Multiphase power flow and state estimation for power distribution systems," IEEE Trans. on Power Syst., vol. 11, no. 2, pp. 939-946, May 1996.

[2] S. Zhong, and A. Abur, "Effects of non-transposed lines and unbalanced loads on state estimation," in Proc. of the PES Winter Meeting IEEE, 2002, vol. 2, pp. 975-979.

[3] I. Dzafic, H.-T. Neisius, and S. Henselmeyer, "Real time distribution system state estimation based on interior point method," Proc. $17^{\text {th }}$ Power Syst. Comp. Conf., Stockholm, Sweden, Aug. 22-26, 2011.

[4] M. E. Baran, and A. W. Kelley, "A branch-current-based state estimation method for distribution systems," IEEE Trans. on Power Syst., vol. 10, no. 1, pp. 483-491, Feb. 1995.

[5] C. N. Lu, J. H. Teng, and W.-H. E. Liu, "Distribution system state estimation," IEEE Trans. on Power Syst., vol. 10, no. 1, pp. 229-240, Feb. 1995.

[6] W.-M. Lin, and J.-H. Teng, "State estimation for distribution systems with zero-injection constraints," IEEE Trans. on Power Syst., vol. 11, no. 1, pp. 518-524, Feb. 1996.

[7] D. A. Haughton, and G. T. Heydt, "A linear state estimation formulation for smart distribution systems," IEEE Trans. on Power Syst., vol. 28, no. 2, pp. 1187-1195, May 2013.

[8] K. Li, "State estimation for power distribution system and measurement impacts," IEEE Trans. on Power Syst., vol. 11, no. 2, pp. 911-916, May 1996.

[9] C.W. Hansen, and A. S. Debs, "Power system state estimation using three-phase models," IEEE Trans. on Power Syst., vol. 10, no. 2, pp. 818-824, May 1995.

[10] M. E. Baran, and A. W. Kelley, "State estimation for real-time monitoring of distribution systems," IEEE Trans. on Power Syst., vol. 9, no. 3, pp. 1601-1609, Aug. 1994.

[11] A. Ghassemian, B. Fardanesh, "Phasor assisted State Estimation for NYS Transmission System - Implementation and Testing", Proc. Power Systems Conference and Exposition, 2009, 15-18 March 2009.

[12] K. D. Jones, J. S. Thorp, R. M. Gardner, "Three-phase linear state estimation using Phasor Measurements," Proc. 2013 IEEE PES GM, 21-25 July 2013.

[13] D. Atanackovic, V. Dabic, "Deployment of real-time state estimator and load flow in BC Hydro DMS - challenges and opportunities," Proc. 2013 IEEE PES GM, 21-25 July 2013.

[14] A. von Meier, D. Culler, A. McEachern, R. Arghandeh, "Microsynchrophasors for distribution systems," 2014 IEEE Innovative Smart Grid Technologies Conference, vol., no., pp.1,5, 19-22 Feb. 2014.

[15] M. Paolone, A. Borghetti, C.A. Nucci, "A synchrophasor estimation algorithm for the monitoring of active distribution networks in steady state and transient conditions," Proc. of the $17^{\text {th }}$ Power Systems Computation Conference, Stockholm, Sweden, Aug. 22-26, 2011.

[16] P. Romano, M. Paolone, "Enhanced Interpolated-DFT for Synchrophasor Estimation in FPGAs: Theory, Implementation, and Validation of a PMU Prototype," IEEE Trans. on Instr. and Meas. (to appear).

[17] Altea Power and Monitoring solutions [Online]. web site: http://www.alteasolutions.eu/.

[18] M. Popovic, M. M. Maaz, D.-C. Tomozei, and J.-Y. Le Boudec. IPRP: "Parallel Redundancy Protocol for IPv6 Networks," Technical report, EPFL, 2014.

[19] L. Zanni, M. Pignati, S. Sarri, R. Cherkaoui, M. Paolone, "Probabilistic assessment of the process-noise covariance matrix of discrete Kalman filter state estimation of active distribution networks," Proc. International Conference of Probabilistic Methods Applied to Power Systems, Durham, UK, Jul. 7-10, 2014

[20] G. Welch and G. Bishop, "An introduction to the Kalman filter," TR 95-041, Dep. of Computer Science, University of North Carolina, USA, July 2006. 